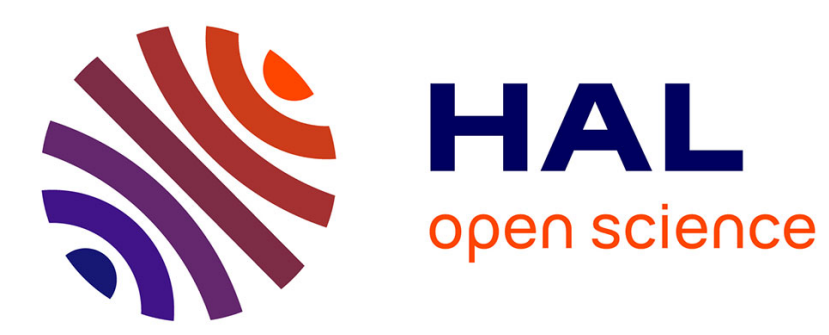

\title{
Collaborer avec le marché : les stratégies des associations végétariennes en France
}

Noé Kabouche, Sophie Dubuisson-Quellier

\section{To cite this version:}

Noé Kabouche, Sophie Dubuisson-Quellier. Collaborer avec le marché : les stratégies des associations végétariennes en France. Revue française de sociologie, 2020, Vol. 61, pp.617-640. 10.3917/rfs.614.0617 . hal-03478654

HAL Id: hal-03478654

https://hal-sciencespo.archives-ouvertes.fr/hal-03478654

Submitted on 14 Dec 2021

HAL is a multi-disciplinary open access archive for the deposit and dissemination of scientific research documents, whether they are published or not. The documents may come from teaching and research institutions in France or abroad, or from public or private research centers.
L'archive ouverte pluridisciplinaire HAL, est destinée au dépôt et à la diffusion de documents scientifiques de niveau recherche, publiés ou non, émanant des établissements d'enseignement et de recherche français ou étrangers, des laboratoires publics ou privés. 


\title{
Collaborer avec le marché : les stratégies des associations végétariennes en France
}

\author{
Noé KABOUCHE et Sophie DUBUISSON-QUELLIER
}

\section{Résumé}

Depuis moins d'une dizaine d'années, la cause végétarienne jouit en France d'une visibilité grandissante au sein de l'espace public, médiatique, ainsi que dans les débats politiques. Cette publicisation de la cause s'accompagne d'une croissance particulièrement rapide du secteur des produits végétariens et véganes sur le marché de l'alimentation. Dans cet article, nous montrons que des organisations du mouvement végétarien contribuent à cette évolution du marché en construisant les conditions d'une collaboration stratégique avec le monde économique, prenant place au sein d'un espace transversal articulant les intérêts des associations militantes et ceux de leurs interlocuteurs marchands. La littérature traitant des liens entre marché et mouvements sociaux a souvent observé les affrontements entre ces deux milieux ou les formes de collaborations mises en œuvre lors de l'élaboration de certifications, valeurs marchandes, ou offres alternatives. Cet article entend contribuer à ces recherches en étudiant les relations directes engagées par les collaborations observées, en soulignant les conditions sociales qui favorisent les interactions entre mondes militant et marchand, ainsi que la porosité des frontières qui délimitent ces espaces.

Mots-clés : végétarisme, collaboration, marché, mouvements sociaux, répertoire d'action collective

La question végétarienne est devenue récemment très visible dans l'espace public ${ }^{1}$. Les médias y accordent une importance croissante ${ }^{2}$, tandis qu'une offre marchande de produits, d'enseignes de restaurants ou de commerce s'est largement développée. Les mouvements végétariens existent en France depuis le $\mathrm{XIX}^{\circ}$ siècle (Traïni, 2011). Ils ont régulièrement contribué à créer ou donner de la visibilité à des offres commerciales (Hondermarck, 2018) pour les adeptes de l'alimentation végétarienne. Aujourd'hui, si la population végétarienne en France reste encore minoritaire (Giroux et Larue, 2017) ${ }^{3}$, le marché des produits végétariens ou

\footnotetext{
${ }^{1}$ Les auteurs souhaitent remercier chaleureusement Philip Balsiger, les membres du comité de lecture de la Revue française de sociologie, ainsi que Laure Bereni pour leurs relectures de l'article. Les propos n'engagent toutefois que les auteurs.

${ }_{2}$ À partir d'une recherche sur l'ensemble de la presse généraliste française, nous observons que le nombre d'articles comportant le nom de la plus célèbre association végane passe de quelques dizaines à environ 1800 , entre 2008 et 2019.

${ }^{3}$ En s'appuyant sur une étude d'OpinionWay de 2016, les auteurs rappellent que 3\% des Français se déclarent végétariens et que $10 \%$ d'entre eux se disent prêts à le devenir. Les données sur la population végane sont en revanche rares.
} 
véganes ${ }^{4}$ connaît une croissance sans précédent ${ }^{5}$ et semble porteur d'importantes promesses financières ${ }^{6}$. Pourquoi le marché s'intéresse-t-il autant à l'alimentation végétale si celle-ci ne constitue encore qu'une demande marginale et est souvent présentée comme engagée, voire militante ? La rapidité de ces évolutions est frappante, si on la compare au cas de l'agriculture biologique, autre forme de consommation marginale et militante, qui a mis près d'une trentaine d’années à connaître de tels développements (Leroux, 2015).

Pour comprendre le processus de développement de ce marché, il est crucial de restituer le rôle joué par les stratégies développées par les associations véganes et végétariennes. Ces associations sont plurielles et ont des positionnements assez hétérogènes. Certaines d'entre elles se situent autour d'un pôle végétarien et mettent en œuvre une démarche experte, qui les conduit à promouvoir une alimentation végétarienne pour améliorer la santé humaine, mais aussi plus récemment à viser des objectifs de protection de l'environnement et de la condition animale, souvent sur un mode pédagogique depuis longtemps emprunté par les acteurs du mouvement (Traïni, 2011, p. 39-62). D'autres, que l'on retrouve, au contraire, davantage au sein d'un pôle végane, sont engagées dans les démarches militantes de la cause animale dénonçant les conditions d'élevage et luttant contre l'abattage des animaux pour l'alimentation ou tout autre besoin humain (Traïni, 2011, p.143-182). L'espace de la cause animale ne recouvre pas celui de la cause végétarienne (Ouédraogo, 2000), notamment parce que certaines associations, dites «welfaristes », agissant pour le bien-être des animaux en élevage ou à l'abattage, ne promeuvent pas l'alimentation végétarienne. En revanche, la plupart des associations promouvant le végétarisme se sont aujourd'hui rapprochées de la cause animale.

\footnotetext{
${ }^{4}$ Le terme « végétarien » qualifie les produits alimentaires ne contenant pas de chair animale. Le terme « végane » désigne des produits qui, en plus d'être végétariens, excluent également toute matière issue d'animaux (œufs, lait, produits de la ruche).

${ }^{5}$ À titre d'exemple, le chiffre d'affaire généré par la vente de produits végétariens et véganes dans les grandes et moyennes surfaces françaises a progressé de 24\% entre 2018 et 2019, selon l'institut d'études Xerfi (janvier 2019). ${ }^{6}$ D'après la banque Barclay, le secteur des alternatives à la viande pourrait peser 140 milliards de dollars dans dix ans, et représenter 10\% du marché de la viande d'ici à 2030. Voir "Le marché de la "viande » vegan promis à une croissance exponentielle", 23 mai 2019, Les Échos.
} 
Nous proposons de montrer dans cet article comment certaines organisations militantes de la cause végétarienne ont développé des stratégies militantes qui ont contribué au développement d'un secteur marchand végétarien et végane ${ }^{7}$, en construisant les conditions d'une collaboration avec le monde économique. Nous soulignons ainsi une forme d'interaction entre mondes militants et mondes économiques, peu analysée dans la littérature, à quelques exceptions près, dans des travaux récents (Buchter, 2020). Si la recherche sur les liens entre marché et mouvements sociaux a connu d'importants développements ces dix dernières années, elle a souvent insisté soit sur l'affrontement entre ces mouvements et les entreprises (Bartley et Child, 2014 ; Dubuisson-Quellier, 2018 ; King et Pearce, 2010), soit sur des démarches de construction de certifications, de valeurs marchandes, ou d'offres alternatives, sans toujours explorer les relations directes engagées par les acteurs militants avec le monde marchand (Bartley, 2007a ; Lounsbury, Ventresca et Hirsch, 2003 ; Dubuisson-Quellier, 2013 ; Balsiger, 2016). Nous voudrions contribuer à cette littérature, en analysant la manière dont des militants choisissent, dans leurs stratégies militantes, de collaborer avec le monde marchand pour les besoins de leur propre cause. En l'occurrence, il s'agit de montrer comment les acteurs de la cause végétarienne cherchent à développer l'offre commerciale végétarienne pour défendre leur cause, en mettant en œuvre un répertoire d'action collective que nous appelons stratégies de «collaboration », au sens de McDonnell et al. (2021). Nous montrons que ce répertoire s'articule avec d'autres répertoires d'action, y compris plus contestataires à l'égard des entreprises et qu'il repose sur la construction de dispositifs marchands (Callon, 2017, p. 395431) ainsi que sur l'existence de porosités sociales et organisationnelles entre les mondes militants et les mondes économiques.

Pour analyse ce mode d'action collaboratif, nous avons enquêté auprès de deux associations situées chacune sur l'un des pôles identifiés plus haut. La première, que nous

\footnotetext{
${ }^{7}$ Pour ne pas alourdir l'écriture, nous utiliserons de manière générique le terme de végétarien, pouvant recouvrir végétarien et/ou végane. Lorsque cela sera nécessaire, nous spécifierons qu’il s’agit de véganisme.
} 
appellerons « Défense animale » (DA), est située sur le pôle végane du mouvement végétarien. Elle est connue pour ses actions très contestataires ayant contribué à donner une forte visibilité publique à la cause de la souffrance animale, par la diffusion de films sur les conditions d'élevage ou d'abattage. La seconde sera nommée « France-Végétarisme » (FV); elle s'inscrit plutôt sur le pôle expert et a pour principal objectif la promotion de l'alimentation végétarienne (cf. Encadré 1).

\section{Encadré 1 : Méthodologie}

France-Végétarisme est une association loi 1901, fondée en 1995. En 2008, ses statuts définissent trois axes principaux : " améliorer la santé humaine, la condition animale, et l'état de l'environnement. ${ }^{8}$ Elle milite ainsi pour la promotion des pratiques de consommation végétale. Pour autant, ses membres revendiquent un objectif de défense de la cause animale s'appuyant sur une conception abolitionniste. FV dispose d'une équipe de 23 membres, dont 3 sont bénévoles et les autres salariés. Elle est financée par les dons et adhésions, par la vente de son magazine trimestriel, Solutions Veggie, ainsi que par les revenus qu'elle tire des certifications VG-Cert attribuées à différentes entreprises. Des partenariats avec des entreprises représentent également une source de revenus, ce qui entraîne une forte dépendance à l'égard du monde marchand. FV mène des actions de plusieurs types visant à accompagner les consommateurs vers une alimentation végétale (ateliers cuisine, pique-niques, recettes, informations nutritionnelles, conférences). Ainsi, cette association "végétarienne » revendique aussi le statut d'association de « défense de la cause animale ».

La deuxième organisation militante est Défense animale (DA). Association loi 1908, DA est fondée en 2008. À la différence de FV qui se positionne plutôt sur un accompagnement doux des consommateurs vers une modification de leurs comportements, DA est spécialisée dans les actions visant à démontrer le bien-fondé supposé de la cause animale, via les stratégies de type «lanceur d'alerte » dans des élevages et abattoirs, souvent illégales, et régulièrement dénoncées par les acteurs pointés du doigt par ces dernières. Néanmoins, ces approches conflictuelles s'accompagnent d'actions pédagogiques, visant à partager recettes véganes et conseils pour une transition vers le véganisme. Enfin, elles s'articulent avec des stratégies de collaboration avec le monde marchand. Le noyau dur

\footnotetext{
${ }^{8}$ Site web de France-Végétarisme.
} 
de DA est composé d'une équipe d'environ 60 salariés et 10 bénévoles. L'association est essentiellement financée par les dons et adhésions, représentant près de $90 \%$ de ses ressources. ${ }^{9}$

Ces associations sont organisées selon des sous-départements spécialisés dans leurs différentes actions reposant essentiellement sur des salariés, dont le travail au sein des associations est souvent une étape au sein d'un parcours professionnel mêlant secteur privé et associatif. Ainsi, pour les actions visant à collaborer avec le monde économique, FV dispose d'une équipe composée de deux principaux salariés, orientés vers la diffusion et la gestion de VG-Cert, un label sur les produits végétariens et/ou véganes. Pour ses initiatives à l'égard du marché, DA compte sur Better, un département spécialisé dans les actions économiques de l'association et géré par une salariée avec un parcours professionnel passé dans la grande distribution. Pour son initiative de référencement de restaurants véganes (RestoVégé), l'association repose sur une autre équipe, coordonnée par cinq salariés, et dépendant de l'action de nombreux bénévoles et adhérents de l'association.

L'enquête a duré sept mois, de l'été 2018 au mois de mars $2019^{10}$. Le recrutement des enquêtés a visé les acteurs centraux des stratégies de collaboration avec le monde marchand.

Au sein des associations DA et FV, nous avons conduit des entretiens semi-directifs auprès des salariés spécifiquement en charge de la coordination des actions menées à l'égard du marché. Ces acteurs, non-bénévoles et ayant parfois un passé dans les entreprises de l'agroalimentaire, se caractérisent par une grande proximité professionnelle avec le monde marchand avec lequel ils dialoguent au cours de leurs actions.

Côté marchand, nous avons mené des entretiens semi-directifs auprès des représentants d'entreprises visées par ces actions. Il s'agit de fondateurs de startups de produits végétariens/véganes, de responsables marketing ou de développement des branches végétariennes/véganes dans de grandes entreprises, et de restaurateurs développant leur offre végane. Ce terrain marchand se compose donc d'acteurs particulièrement proches et informés des enjeux liés au végétarisme, au véganisme, et des actions militantes élaborées par les associations.

Au total, 27 entretiens ont été conduits.

Plusieurs observations directes ont été menées lors d'événements en lien avec le véganisme. Ces dernières ont été utiles dans l'étude de l'espace des actions collaboratives traversant les mondes marchands et la sphère militante.

\footnotetext{
${ }^{9}$ Site web de DA.

${ }^{10}$ L'enquête a été conduite par Noé Kabouche dans le cadre de son mémoire de master.
} 
Enfin, un large panel de sources écrites a été examiné. Du côté des entreprises, des documents de communication externe (notamment des publicités) ont été consultés. Côté associations militantes, nous avons été attentifs aux documents de communication à destination des sympathisants, adhérents des associations en question (revues, tracts, prospectus) mais aussi à destination du grand public (vidéos, articles sur les sites web). Des documents internes ont également été analysés (contrats, chartes, cahiers des charges, diaporamas PowerPoint).

Les noms des individus, des organisations et des initiatives portées par celles-ci sont anonymisés, sauf si le contraire est indiqué, en particulier dans le cas où le nom réfère à un acteur externe à l'enquête de terrain, et que nous estimons que l'absence d'anonymisation apporte des données de contextualisation utiles.

Dans la première partie de cet article, nous présenterons le mode d'action collaboratif déployé par les deux associations de la cause végétarienne, en montrant que leur caractère militant tient aux usages stratégiques qu'en font les associations pour orienter les entreprises vers le développement de l'alimentation végétarienne. Dans la seconde partie, nous interrogerons les conditions sociales qui rendent possibles cette collaboration stratégiques en insistant sur les porosités entre ces deux mondes sociaux, que sont les sphères marchande et militante.

\section{Singulariser l'offre afin d'en faire un levier pour la cause végétarienne}

La littérature étudiant les relations entre marchés et mouvements sociaux a souvent insisté sur les démarches déployées par certains de ces mouvements pour développer des labels ou des certifications - comme dans le cas du commerce équitable — dont les entreprises peuvent ensuite se saisir pour singulariser leur offre (Le Velly, 2006). Elle a pu analyser les tensions que pouvaient provoquer la construction ou la gestion de ces certifications, entre les différentes parties prenantes engagées dans la gouvernance (Barral, 2015, p. 185-208 ; Bartley, 2007a). Mais elle s'est moins intéressée aux conditions concrètes dans lesquelles des 
organisations militantes et des entreprises pouvaient interagir autour de ces dispositifs marchands. En suivant les démarches de nos deux associations, DA et FV, dans le développement de différents dispositifs marchands avec des restaurants et des industriels, nous pouvons analyser la manière dont ces dispositifs leur permettent de faire de l'offre un véritable outil de la cause végétarienne.

Nous commencerons par étudier deux actions visant les restaurants, menées par l'association Défense animale (DA) : RestoVégé et Better. Puis nous examinerons le label VGCert, géré par France-Végétarisme (FV), et proposé aux industriels de l'agroalimentaire. Nous montrerons alors comment les organisations font des usages variés et modulés de ces instruments pour trouver des leviers pouvant articuler sanction et récompense, afin d'amener les acteurs économiques à développer leur offre végétarienne.

\section{Classement et référencement : des outils pour les restaurants}

En tant qu'acteurs centraux du marché alimentaire, les restaurants constituent un maillon important pour DA. L'association propose alors gratuitement aux restaurateurs des outils de singularisation de leur offre, comptant sur les effets de levier liés à la recherche de distinction et à la concurrence propre à ce secteur pour donner de la visibilité à la cause végétarienne. Ces instruments marchands ont la particularité d'être étroitement liés aux répertoires militants, qu'il s'agisse d'articuler sanction et récompense, ou de mobiliser des consommateurs pour organiser une consommation engagée.

L'évaluation des restaurants est un dispositif mis en place par Better, un département de l'association DA, créé en février 2018 et dédié à l'ensemble des actions de DA agissant sur le marché. L'une des initiatives de Better consiste à classer les grandes chaînes de restauration françaises (telles que Starbucks, Subway, Paul, McDonald's...) : plus l'offre végane des 
restaurants est vaste, meilleure est la note qui leur est attribuée. Le classement est ensuite disponible en ligne sur le site de DA et peut être consulté par les consommateurs. Carine, âgée de 40 ans, et salariée de DA, est chargée des différentes étapes du référencement des menus, des établissements, et de l'élaboration de la méthodologie de l'évaluation. Cette dernière prend principalement en compte le nombre de plats véganes proposés et la diversité de l'offre, en examinant si elle concerne aussi bien les entrées, que les desserts et les plats principaux.

L'autre outil, RestoVégé, est également porté par DA, depuis 2015. Il consiste à référencer en ligne les restaurants français qui proposent des plats véganes. Ces établissements ne sont pas des chaînes et peuvent être de très petites entreprises ; c'est donc un autre type d'acteur qui est ici visé. Par ailleurs, le dispositif donne la main aussi aux clients eux-mêmes, soucieux de pouvoir trouver une offre adaptée, mais aussi aux militants actifs de la cause, puisque n'importe quel utilisateur de l'application ou du site RestoVégé peut proposer à DA un restaurant à référencer. Les bénévoles de l'association se chargent ensuite de contacter les restaurateurs, tandis que des salariés de DA sont affectés à la gestion du site web, du suivi des restaurateurs, et de la communication.

Ces deux dispositifs correspondent à ce que Lucien Karpik $(2000,2007)$ a défini comme des dispositifs de jugement, destinés à guider les choix des consommateurs. Ils s’inscrivent dans un contexte où d'autres dispositifs de ce type existent déjà, notamment pour les restaurants, comme le guide HappyCow ${ }^{11}$ créé en 1999 et évaluant les restaurants végétariens et/ou véganes dans le monde entier. Le système RestoVégé fonctionne sur un système de singularisation qui distingue parmi l'offre de restaurants ceux qui bénéficient du référencement de l'association, tandis que Better articule cette singularisation avec un système de classement, puisque, parmi les restaurants qui sont référencés, certains peuvent avoir une meilleure évaluation que d'autres.

\footnotetext{
${ }^{11}$ Non anonymisé.
} 
Mais si la littérature de sociologie économique a souvent examiné les effets sur les consommateurs (Cochoy, 2002), elle a aussi insisté sur la manière dont ces dispositifs pouvaient modifier les conduites des entreprises (Barraud de Lagerie, 2006, 2009 ; Dubuisson-Quellier, 2017). Il s'agit en effet pour l'association de contribuer au " développement de l'offre végane » et de "rendre l'alternative végétale disponible pour le plus grand nombre » (Carine, 40 ans, DA), au nom de la cause végétarienne. L'instrument marchand devient ainsi un élément du répertoire militant. L'association va alors jouer sur les différents usages possibles de l'outil dans la collaboration pour inciter les entreprises à adopter les pratiques les plus favorables à la cause végétarienne. Dans le cas de Better, l'outil, parce qu'il permet de classer et donc de comparer les offres entre elles (Espeland, 1998), peut dans un premier temps être utilisé sur un mode punitif, et donc plutôt contestataire. Comme l'explique Carine, la responsable de Better, attribuer de mauvaises notes à des chaînes de restauration pour sanctionner le fait qu'elles font peu de place à une offre végétarienne est un moyen d'exercer une pression sur elles. Les classements agissent comme des éléments du répertoire de naming and shaming (Bartley et Child, 2014) au sens où ils contribuent à rendre publiques des sanctions de certaines pratiques des entreprises. Comme l'indiquent ces deux auteurs, le fait de cibler des enseignes de la restauration à forte notoriété permet de gagner en visibilité et en efficacité. Mais ces actions de naming and shaming, inscrites dans le répertoire contestataire, sont également un moyen d'aller plus loin, et y compris de parvenir à une collaboration avec l'entreprise. Ainsi, au cours de l'élaboration des classements, l'association DA a informé chacune des entreprises de sa démarche :

« Quand j’avais commencé à faire cette opération de classement, (...) j’ai envoyé un mail à ces chaînes (...) : 'J'ai relevé ça ; et je vous le soumets, et vous êtes libres de ne pas me répondre, ce qui ne sera pas forcément en votre faveur, puisque j'aurais pu omettre une offre que vous proposez et que je n'ai pas vue.' » (Carine, 40 ans, responsable de l'initiative Better, DA) 
La critique ouverte adressée aux entreprises est alors utilisée comme un moyen d'entrer en contact, d'ouvrir une séquence d'interaction, pour engager une négociation, voire une collaboration, comme dans le cas d'instruments de naming and shaming (Dubuisson-Quellier, 2020). Cette discussion avec les entreprises permet à DA d'utiliser les arguments prisés par les entreprises, comme la différenciation stratégique et la réputation, afin de les inciter à développer une offre végétarienne. DA compte ainsi mettre en scène des dynamiques de concurrence entre les entreprises et y introduire le rôle joué par une offre végétarienne :

« On sait que si on appuie sur le bouton 'concurrence', y'en a qui voudront être en avance par rapport aux autres, et là c'est un peu ce qui s'est passé » (Carine, 40 ans, responsable de l'initiative Better, DA).

Une fois le dialogue ouvert, l'association cherche à utiliser l'instrument dans un logique de gratification, en accompagnant les entreprises dans leurs efforts de végétalisation de l'offre.

« On y va avec un chef. Et puis on voit par rapport (...) à leur offre, ce qu'on peut leur proposer, les endroits où ils s'approvisionnent, ce que leur chef connait ; on essaye de discuter pour voir vers quoi on peut aller, en fonction aussi du style de restauration proposé. » (Carine, 40 ans, responsable de l'initiative Better, DA)

L'accompagnement consiste ainsi dans des rencontres et discussions où les acteurs de DA mettent en avant les possibilités d'évolutions véganes les plus adaptées aux attentes des consommateurs, en leur recommandant des produits conformes à l'identité du restaurant. Audelà de la sélection de nouveaux produits de base, le fait de s'appuyer sur la présence d'un chef lors de ces rendez-vous permet également d'aller plus loin en travaillant à l'élaboration de nouvelles recettes. Ensuite, DA propose une publicité de ces restaurants au sein de la presse professionnelle :

«Pour susciter un peu l'envie et la jalousie chez les autres, [on va y dire des choses comme] : 'ah bah tiens, eux ils ont fait des efforts, ils sont sensibles à la condition animale, c'est une chaîne dynamique.' » (Carine, 40 ans, responsable de l'initiative Better, DA)

L’instrument RestoVégé fonctionne différemment. Il ne repose pas sur un classement des offres, mais sur un signal qui permet de singulariser le restaurant en indiquant que son offre 
végane est approuvée par l'association DA. Comme indiqué plus haut, les consommateurs végétariens peuvent signaler les restaurants à l'association, qui cherche à maximiser le nombre de référencements, pour rendre visible le dispositif, et inciter le plus de restaurants possibles à chercher à se faire référencer. Cependant, cette démarche de labellisation est articulée à un dispositif du répertoire militant en matière de consumérisme politique (Dubuisson-Quellier, 2018) appelé le Carrot'Mob, dans lequel des militants vont organiser une action consistant à faire venir des consommateurs chez un commerçant pour l'inciter à apporter des améliorations en faveur d'une cause. Le terme, formé des mots « mobilisation » et « carotte », par opposition au bâton, insiste sur la récompense associée à l'action. Hoffmann et Hutter (2012) le définissent comme « un buycott ${ }^{12}$ ponctuel (...) organisé par des militants, (...) qui sélectionnent pour cela une entreprise ayant formulé (...) une offre correspondant aux (...) attentes et/ou aux demandes des militants. » (p. 218, notre traduction) Ainsi, DA organise ce qu'elle nomme des « défis » lancés par les bénévoles de l'association à des restaurateurs qui ne sont pas encore référencés. Un « défi RestoVégé » consiste, pour le restaurant, à proposer une soirée entièrement végane, avec des menus et des prix élaborés en concertation avec DA. Si le restaurant accepte, alors DA se charge de remplir la salle, en organisant une importante communication via ses réseaux, et les plats sont évalués par les clients à la fin de la soirée. Cette stratégie vise à inciter le restaurant à mettre à sa carte de manière permanente un ou des plats véganes qui ont remporté l'adhésion, et ainsi obtenir le référencement RestoVégé :

« On va voir les restaurateurs, et on leur dit : 'est-ce que ça vous tente de nous faire un menu 100\% végane le temps d'un repas ?' Et nous, en échange, on remplit la salle de restauration, on gère les réservations, et comme ça, le cuistot, il est tranquille, il peut tester ses recettes et il sait qu'il y’a du monde dans la salle : pas de risque. Et nous, ensuite, on fait remplir un questionnaire aux convives pour savoir comment ils ont trouvé le menu, et après on fait un retour au restaurateur. (...) Et après, on voit avec le resto si ça le tente de garder le menu. » (Aurélie, 32 ans, responsable de la gestion des référencements RestoVégé, DA)

\footnotetext{
${ }^{12}$ Le néologisme "buycott" renvoie à une action menée par les consommateurs consistant à récompenser les pratiques vertueuses d'une entreprise en consommant massivement chez elle.
} 
Le dispositif cherche ainsi à faire d'une offre végétarienne un moyen par lequel un restaurant peut accroître sa visibilité, voire gagner en notoriété. Il contribue aussi à rendre plus visible, par le marché, la cause végétarienne dans l'espace public.

Comme dans le cas de Better, ce processus de collaboration stratégique se poursuit à travers un accompagnement et un soutien offerts aux restaurateurs dans leur démarche de végétalisation de l'offre. Par exemple, les responsables de l'initiative RestoVégé accompagnent les restaurants référencés en les mettant en relation avec des fournisseurs de produits véganes :

« Généralement, on les appelle soit parce qu'on les a vus sur les salons (...) soit [grâce à notre] veille médias. Après, (...) dans la newsletter qu'on envoie aux restaurateurs, on leur dit les fournisseurs qu'on a référencés, et on les tient au courant des nouveautés. » (Aurélie, 32 ans, responsable de la gestion des référencements RestoVégé, DA)

Sur le site RestoVégé, dans la section destinée aux restaurateurs, on peut également trouver une liste de 38 marques véganes, auprès desquelles il est possible de se fournir. DA agit ici en mettant à leur disposition des ressources informationnelles destinées à faciliter leur transition vers une offre végétale.

Ainsi, via des actions collaboratives permettant de viser des acteurs-clés de l'offre alimentaire, DA mène une stratégie de végétalisation de l'offre auprès des restaurants, se traduisant par des collaborations ponctuelles, et parfois adossées à des premières démarches volontairement contestataires, visant à engager les enseignes de restaurants à réagir. Nous verrons que l'association FV, avec VG-Cert, opte pour une stratégie reposant sur des objectifs semblables, mais sur un usage sensiblement différent, impliquant notamment des collaborations plus profondes.

\section{Label et prestations de service : les outils pour l'industrie agroalimentaire}


VG-Cert est dispositif de labellisation des produits végétariens ou véganes issus de l'industrie agroalimentaire. Il permet de distinguer le produit par un symbole présent sur l'emballage, destiné à indiquer qu'il a été élaboré avec des ingrédients et dans des conditions qui respectent les critères végétariens ou véganes. Ce label, géré en France par l'association France-Végétarisme (FV), permet à cette dernière de développer des liens de collaboration forts avec des acteurs agroalimentaires et d'accompagner ceux-ci vers la végétalisation de leur offre. FV est en réalité l'opérateur français de ce label, qui est européen. L'idée du label VG-Cert naît dans les années 1990 en Suisse, puis le label est officiellement lancé dans toute l'Europe en 1996 par l'association EVB (European Vegetarian Band). Il s'agit d'un consortium d'associations végétariennes et véganes européennes, constitué de 36 membres. La marque et le logo VG-Cert sont officiellement coordonnés et contrôlés par l'EVB, mais la délivrance de VG-Cert dans chaque pays européen est déléguée à une association nationale. FV en a la gestion en France depuis 2015. Une dizaine de labels végétariens ou véganes dont les agences sont basées en Europe peuvent être retrouvés sur les produits commercialisés en France, mais VGCert, qui se décline en deux versions, «Végétarien » et « Végane », est celui qui référence le plus grand nombre de produits - environ 800 - sur le territoire. Agathe (24 ans, responsable VG-Cert) et ses collègues ont la charge de gérer et développer ce label pour l'association. Ils réalisent une veille médiatique et commerciale des produits ou entreprises qui pourraient répondre aux critères du cahier des charges, mais procèdent aussi par des sollicitations et démarches actives, lors des salons professionnels. Enfin, ils peuvent répondre à des demandes formulées par les entreprises qui souhaitent obtenir ce label pour un produit. Les marques ciblées par ce label sont très diverses : il peut s'agir de startups développant des produits véganes, d'entreprises moyennes fournissant les magasins bios, ainsi que de très grands groupes, industriels ou distributeurs, développant, au sein de départements spécialisés — à côté de leurs gammes non-végétales —, de nouveaux produits végétariens ou véganes (Danone, 
Herta, Carrefour...). À la différence des actions menées par DA, ces services de labellisation ne sont pas gratuits : l'usage du label par les entreprises est concédé moyennant le paiement d'une redevance annuelle allant de 30 à 350 euros par produit labellisé, selon le nombre de produits et la taille de l'entreprise ${ }^{13}$. Ces entrées d'argent liées à la labellisation mais aussi aux services de promotion vendus aux entreprises (que nous présenterons plus loin) représentent près de $30 \%$ des revenus de $\mathrm{FV}$, soit une part considérable des ressources de l'association. Ces évolutions de FV sont caractéristiques des mutations récentes connues par le secteur associatif, qui a non seulement diversifié ses ressources à travers des activités économiques de prestation de services, mais aussi davantage recruté des profils de salariés formés à la recherche et la gestion de ces nouvelles ressources économiques (Simonet, 2012).

Comme dans les cas de Better et de RestoVégé, on comprend que, si VG-Cert est bien un dispositif de jugement pour le consommateur, ce dernier n'en est pas la cible immédiate : l'objectif est avant tout de modifier l'offre. Pour cette raison, le système de label est fortement articulé avec des activités de prestation de service qui l'accompagnent, et qui sont destinées à transformer l'offre. Ces activités sont particulièrement développées dans le cas de FV. Ces prestations de service sont toutefois au service de la cause végétarienne défendue par FV, car elles se fondent sur le principe selon lequel, plus l'alimentation végétarienne sera accessible et visible dans l'espace public, plus elle sera susceptible de se développer, ce qui suppose que les entreprises considèrent qu'elles peuvent y avoir intérêt :

«L'idée c'est de leur donner de la reconnaissance, de la visibilité et du coup de générer l'émulation (...) auprès des autres entreprises, qui se diront : 'ah bah tiens, là y'a quand même un créneau pour améliorer son image, travailler le côté $\mathrm{RP}^{14}$ '. » (Agathe, 24 ans, Responsable VG-Cert, FV)

Ces actions de prestation de service ne constituent donc pas un moyen complémentaire et annexe d'accroître les ressources de l'association. Comme les services proposés aux

\footnotetext{
${ }^{13} \mathrm{FV}$ pratique des tarifs dégressifs, et des prix plus avantageux pour de petites entreprises.

${ }^{14}$ Relations publiques.
} 
entreprises, par les associations engagées dans la promotion de la diversité en entreprise, pour se mettre en conformité avec le droit, étudies par Lisa Buchter (2020), ou encore les services de compensation carbone vendues aux entreprises pour les inciter à évaluer et contrôler leurs émissions de gaz à effet de serre, étudiés par Alice Valiergue (2021), les prestations de services vendues pas FV font partie du répertoire de l'action militante de la cause végétarienne.

Pour FV, le label est un outil à multiple détente. Il permet d'ouvrir le dialogue avec les entreprises et d'orienter leurs choix en leur fournissant des solutions concrètes pour développer une offre végétarienne :

«L'effet direct du label est pas si important que ça (...). Mais (...) on va pousser les entreprises à développer leur gamme veggie et (...) et on va créer des contacts. On va aussi initier des changements chez les grandes entreprises qui vont aussi se poser la question 'pourquoi pas développer une gamme veggie, végane etc. ?' et ça nous permet (...) d'entrer en contact avec d'autres acteurs économiques. » (Agathe, 24 ans, responsable VG-Cert, FV)

Les entreprises viennent y chercher non seulement des services techniques et de marketing, mais aussi la légitimité dont bénéficie FV dans le champ du végétarisme. L'association sait qu'elle ne vend pas seulement des prestations, mais qu'elle donne aux entreprises l'accès à des ressources symboliques susceptibles d'exciter la concurrence entre elles, et de favoriser le développement accéléré de l'offre. Au terme de ce processus, l'association gagne des revenus, mais surtout, la cause progresse.

FV doit d'abord convaincre les entreprises de l'intérêt qu'elles auraient à développer ou approfondir des gammes végétales. Pour cela, l'association organise des conférences privées à destination des entreprises, au cours desquelles elle présente des études de marché, des données chiffrées sur la croissance du marché et sur les évolutions des comportements de consommation ainsi que les effets positifs possibles sur l'image de l'entreprise. La démarche de FV peut ainsi être assimilée un travail de construction sociale du marché (Steiner, 2005 ; Garcia-Parpet, 2008 ; Zelizer, 1978). Souvent produit par les acteurs économiques eux-mêmes, ce travail n'exclut pas la participation d'autres acteurs, comme les acteurs publics (Dubuisson-Quellier, 
2016, p. 193-228), ou, dans le présent cas, des acteurs militants (Dubuisson-Quellier, 2013) qui vont suggérer l'existence d'une demande, non pas pour vendre leurs produits, mais pour inciter les entreprises à développer une offre.

Lorsque le contact avec les entreprises a été ainsi établi, FV propose plusieurs types de services, qui ne sont pas facturés en plus de la redevance liée au label :

«L'accompagnement [des entreprises], c'est une équipe dédiée sur Paris, à l'écoute, qui est là non seulement pour les conseils sur le label, mais également sur toutes les questions végétales. » (PierreEmmanuel, 40 ans, responsable VG-Cert et relations avec les entreprises, FV)

Trois types d'actions sont proposés pour engager les entreprises, dans ce qui est présenté par l'association comme une végétalisation du marché, et en tirer pleinement partie, commercialement. FV fournit des services juridiques : l'association se tient informée des évolutions des lois concernant les substituts végétaux et des tendances de ces dernières. Elle répond aux questions des entreprises sur les termes à employer (par exemple : 'steak', 'lait', 'végétal', 'veggie', 'végane', etc.) afin d'éviter tout litige juridique. Deuxièmement, elle se présente comme un intermédiaire capable de faire le lien avec le milieu associatif, renseignant les entreprises labellisées sur les attentes des communautés militantes, mais aussi les risques liés aux éventuelles actions militantes auxquelles pourraient s'exposer les entreprises si elles ne se conforment pas à ces attentes. Troisièmement, FV propose des services techniques aux entreprises, en les aidant à trouver les matières premières, les fournisseurs, les solutions techniques, en participant à des évaluations de la qualité des produits. Cet accompagnement partenarial offre ainsi à l'association des moyens relativement nombreux de faire avancer vers une offre végétarienne les entreprises bénéficiant de ces services.

Pour bien comprendre comment ces dispositifs collaboratifs s'inscrivent dans un registre militant, il convient de considérer la manière dont ils s'articulent à des actions plus contestataires ciblant le monde économique, menées par d'autres organisations engagées dans la cause végétarienne. Pierre-Emmanuel, l'un des salariés de FV en charge de VG-Cert, rappelle 
qu'une partie de l'efficacité des actions collaboratives tient au fait que d'autres organisations de la cause végétarienne, notamment $\mathrm{DA}$, mènent des actions plus contestataires qui contribuent non seulement à rendre visible la cause, mais aussi à faire émerger une menace et donc des risques pour les entreprises :

« DA va vraiment être là pour opérer comme un choc, une espèce d'éveil des consciences pour dire 'regardez ce qui se passe' ${ }^{15}$. À partir du moment où (...) les gens sont alertés là-dessus, notre rôle c'est de les accompagner, leur dire : 'vous avez vu ce qui se passe, ne vous inquiétez pas, tout va bien se passer ; on va vous accompagner dans le changement' (...) à travers VG-Cert, en disant 'regardez, y'a plein de produits qui sont labellisés aujourd'hui, c'est extrêmement facile'. (Pierre-Emmanuel, 40 ans, FV)

Ces menaces et ces risques sont bien perçus par les entreprises, comme l'une d'entre elles nous l'a confirmé. Craignant une action de dénonciation des conditions d'élevage et d'abattage des animaux, menée par DA, l'[Entreprise X] s'est tournée vers FV, sur les conseils de DA, pour s'engager dans une démarche de développement d'une gamme de produits, labellisée VG-Cert :

«DA m'a mis en contact avec France-Végétarisme (FV). On a vu qu'il y avait une passerelle, justement, entre les deux. Et puis que FV, elle a été... peut-être... bah, militante, mais pour l'alimentation végétarienne, mais moins 'trash' (...), plus modérée. » (Baptiste, 50 ans, responsable agriculture et qualité, [Entreprise $\mathrm{X}])$

Au sein de la cause végétarienne, des tensions peuvent exister entre les différents acteurs militants du fait de leurs positionnements différents ; mais ceux-ci travaillent aussi ensemble, pour faire progresser leur cause. Certaines associations font ainsi émerger des risques pour les entreprises auxquelles d'autres - parfois les mêmes, car nous avons vu que DA jouait sur les deux types d'action - apportent des solutions. Cette division du travail est recherchée et revendiquée, comme l'indique Carine (DA) qui affirme que les deux associations « collaborent et se partagent le travail », afin «d'avancer conjointement », y compris dans le cadre d'autres initiatives, mais aussi avec d'autres organisations, parfois plus radicales, ou au contraire plutôt

\footnotetext{
${ }^{15}$ Pierre-Emmanuel fait ici référence aux campagnes de DA dénonçant les marques se fournissant auprès d'élevages pointés du doigt pour mauvais traitement des animaux.
} 
positionnées sur le pôle modéré ${ }^{16}$, welfariste ${ }^{17}$. Comme le souligne Pierre-Emmanuel, les entreprises ont une lecture assez lucide des positionnements des différentes organisations et font elles-mêmes un usage relativement stratégique des ressources que les unes ou les autres peuvent fournir :

« Les grandes [entreprises], (...) elles sont en général assez demandeuses : (...) on a une image qui est assez verte, assez cool, assez docile, donc pour elles... elles sont assez demandeuses. » (Pierre-Emmanuel, FV)

A ces positionnements différenciés des associations, fait écho également une structuration segmentée du monde économique, sur laquelle les associations jouent aussi. En effet, FV travaille aussi bien avec des grands groupes qui permettent de donner une visibilité aux labels et entraîner d'autres entreprises à suivre cette voie, qu'avec des startups jouant le rôle de challengers (Fligstein et McAdam, 2011), par des démarches innovantes en matière d'offre végétarienne, et contribuant ainsi à tirer le marché vers le développement de l’offre.

Au terme de cette présentation, nous avons mis en évidence un espace social très hybride, dans lequel les différentes organisations économiques et militantes interagissent. D’une part, les organisations militantes développent un usage stratégique de leurs positionnements relatifs, mais aussi des positionnements des entreprises. D'autre part, les collaborations stratégiques s'articulent avec des actions plus frontales et contestataires, qu'elles soient portées par des organisations militantes différentes ou parfois par les mêmes organisations. Nous avons insisté sur les dimensions militantes de ces démarches partenariales précisément parce qu'elles s'articulaient à des actions de portée plus contestataire, parce qu'elles visaient les objectifs de la cause végétarienne de l'association, et enfin parce qu'elles s'appuyaient sur un usage

\footnotetext{
${ }^{16}$ Ces stratégies de collaborations ne produisent cependant pas que des synergies : les actions collaboratives peuvent parfois être utilisées très ponctuellement par les entreprises, sans que celles-ci apportent des modifications structurelles à leurs gammes. Ainsi, en offrant aux entreprises une " porte de sortie » face aux approches frontales, ces actions peuvent aussi fragiliser l'efficacité des approches conflictuelles, comme le montre Balsiger (2014).

${ }^{17}$ Voir note 6, p. 2.
} 
stratégique des positionnements différentiels des organisations engagées dans la cause et des entreprises.

Cette réalité nous conduit à envisager les importantes porosités qui existent entre ces espaces sociaux, notamment entre les milieux associatifs et les mondes économiques. Nous avons noté que les associations n'ont pas de difficulté à entrer en contact avec les entreprises, qu'elles pouvaient organiser des conférences à destination de ces dernières et proposer des services d'accompagnement assez facilement. Nous avons donc souhaité interroger les conditions sociales d'une telle porosité pour mieux comprendre comment mondes militants et mondes économiques pouvaient se rencontrer, interagir et même collaborer. Ce sera l'objet de notre deuxième partie, explicitant ce qui rend possible le déploiement de ces modes d'action collaboratifs.

\section{Les porosités entre mondes marchand et militant comme conditions}

\section{sociales de la collaboration stratégique}

Malgré l'accent mis par la littérature sur les conflictualités entre les mondes militant et marchand, certains travaux soulignent leur convergence d'intérêts et leur collaboration dans le processus de développement de labels et de certifications (Bartley, 2003, 2007b) ; d'autres montrent comment des initiatives militantes contribuent au développement d'opportunités économiques pour les acteurs marchands (Hiatt, Sine et Tolbert, 2009 ; Weber, Heinze et DeSoucey, 2008). Néanmoins, peu de travaux ont directement interrogé les conditions sociales qui rendaient possibles ces collaborations. Nous montrons que, dans notre cas, les salariés d'associations militantes étudiés disposent, à la manière des entrepreneurs de cause (Neveu, 2011), de ressources symboliques et matérielles leur permettant d'activer leur cause sur le marché de l'alimentation, en l'articulant assez aisément avec les intérêts économiques de ce secteur. Nous ne pouvons cependant pas parler d'un espace de la cause (Bereni, 2019) qui 
inclurait à la fois ces mondes militants et ces mondes marchands, car, au fond, il n'est pas nécessaire que les acteurs en entreprise soient eux-mêmes militants, ou même adhérents à la cause pour qu'ils travaillent en faveur d'une offre végétarienne, même si certains d'entre eux peuvent être végétariens. Ce qui nous semble bien plus significatif, dans notre cas, est le fait que les acteurs du monde militant disposent des ressources pour articuler les objectifs de la cause végétarienne avec des objectifs économiques des entreprises : l'offre végétarienne est pour les militants un moyen de développer et de promouvoir l'alimentation végétarienne, et elle est, pour les acteurs économiques, une nouvelle source d'opportunités commerciales. Par conséquent, même si, parfois, la convergence d'intérêts et d'idéologies reste possible — comme dans le cas d'un restaurateur militant pour cause végétarienne qui travaille pour être référencé — elle n'est pas indispensable à la collaboration, chacune des deux parties pouvant continuer à viser ses propres intérêts. Cependant, les porosités sont importantes entre ces acteurs associatifs et ceux des entreprises, et nous proposons de les repérer de deux façons : d'une part, en examinant les appartenances et les trajectoires sociales semblables qui peuvent faciliter les rencontres mais aussi les circulations entre ces deux mondes ; d'autre part, en analysant les échanges de ressources économiques et symboliques qui favorisent les convergences entre les acteurs, sans pour autant nécessiter la convergence de leurs intérêts.

\section{Des trajectoires militantes, professionnelles et interpersonnelles croisées}

Les salariés d'associations militantes et les acteurs marchands prenant part aux stratégies de collaboration ont sensiblement le même âge et les même parcours scolaires et universitaires.

D'abord, la plupart des enquêtés sont jeunes. Parmi les salariés d'associations militantes, aucun n'a plus de 40 ans. Et, à titre d'exemple, seuls 3 des 9 représentants 
d'entreprises ${ }^{18}$ enquêtés ont plus de 40 ans. Plusieurs enquêtés sont très jeunes comme Agathe (FV), 24 ans ou Chloé (cofondatrice de Veggie'z, entreprise partenaire de FV), 25 ans. Au-delà de l'âge, les enquêtés sont issus des mêmes milieux sociaux, que l'on pourrait qualifier de CSP ++ , et partagent des parcours scolaires et professionnels similaires. Ils ont en général un haut niveau d'études (master ou doctorat) et certains sont issus des grandes écoles. C'est particulièrement le cas chez les acteurs en entreprises qui interagissent avec les acteurs associatifs. Ainsi, Chloé (Veggie'z, entreprise partenaire de FV) est diplômée de Sciences Po Paris, en master communication ; Aude (responsable Iltro France, entreprise de produits alternatifs aux produits laitiers, certifié VG-Cert) est diplômée d'HEC Paris ; Corentin (responsable communication d'une entreprise de sauces végétales, certifiée VG-Cert) détient un master d'école de commerce ; Flavien (cofondateur d'une startup de simili-carnés, certifiée VG-Cert) est ingénieur en agronomie. En plus d'être très diplômés, ces acteurs, dans la plupart des cas, ont un parcours en lien avec la communication ou le commerce. Mais c'est aussi le cas des acteurs associatifs : Agathe (responsable VG-Cert à FV) est diplômée de l'IEP de Rennes, en politiques alimentaires ; Pierre-Emmanuel (responsable VG-Cert à FV) est issu d'une école de publicité, et détient un master d'école de commerce ; Carine (responsable Better à DA) a un parcours marketing dans l'agroalimentaire et la restauration commerciale. Ainsi, salariés d'associations et représentants d'entreprises, de par leur formation, mais aussi leurs expériences professionnelles pour certains, appartiennent aux milieux professionnels du commerce, de la restauration, de l'agroalimentaire et de la communication.

Les trajectoires professionnelles des salariés des associations en charge de ces actions de collaboration frappent par leur similarité avec celles de leurs homologues en entreprise. Ainsi, à FV comme à DA, ces salariés, membres d'une " entreprise associative » couplant contrat d'association et contrat de travail (Hély, 2004), ont eux-mêmes travaillé par le passé

\footnotetext{
${ }^{18}$ Hors restaurants.
} 
dans des entreprises de l'alimentaire. Dans ce cadre-là, leurs pratiques militantes peuvent être reconverties en opportunités professionnelles, et vice-versa (Simonet, 2012). Ainsi, Carine rejoint DA après un parcours dans le marketing et l'agroalimentaire; par le passé, elle accomplissait le travail que réalisent aujourd'hui ses interlocuteurs marchands. Cela n'a bien sûr rien d'un hasard et c'est bien pour son expérience en entreprise que DA la recrute :

« J'ai travaillé près de quinze ans dans l'agroalimentaire ; j'ai travaillé chez des gros faiseurs (...). Et puis après, j'ai travaillé en direction marketing pour une chaîne de restaurants. (...) Et j'ai commencé par devenir végétarienne à force d'entendre des scandales alimentaires, etc. (...) et puis (...) j'ai lu Faut-il manger les animaux $^{19}$ ? Et le coup de grâce, c'est quand j'ai vu Cowspiracy ${ }^{20}$. (...) Et je suis rentrée chez DA au mois de février [2018], [après avoir] postulé à une offre. Et c'est le parcours que j'ai dans l'agroalimentaire qui a convaincu. » (Carine, 40 ans, responsable de l'initiative Better, DA)

La trajectoire militante s'articule ici à la trajectoire professionnelle et n'en est pas indépendante (Agrikoliansky, 2017 ; Fillieule, 2001). Carine incarne ainsi ces circulations entre les espaces marchands et militants, puisqu'elle travaille pour une association militante, mais en collaboration avec le monde économique dont elle est elle-même issue. Pierre-Emmanuel (FV) présente une carrière similaire, mais plutôt dans les secteurs du commerce et de la communication :

« Je viens d'une école de pub, j'ai fait un master pour une école de commerce, et après, j'ai fait toute ma carrière en presse. (...) Et du coup, moi, mes compétences étaient essentiellement commerciales.» (Pierre-Emmanuel, 40 ans, FV)

Il ajoute qu'il a travaillé dans des grands groupes de presse, indiquant qu'il a « $d u$ mal à s'exprimer aujourd'hui » sur ces derniers, et sur cette période de sa carrière professionnelle, faisant ainsi de ce parcours très commercial un stigmate, tout en le transformant en atout, en indiquant que ce parcours lui a permis de constituer un "carnet d'adresses " utile pour la promotion de l'activité militante de FV ou en estimant que, par son parcours dans la

\footnotetext{
${ }^{19}$ Il s'agit d'un ouvrage de Jonathan Safran Foer, plaidoyer pour le végétarisme.

${ }^{20}$ Il s'agit d'un film documentaire de Kip Andersen et Keegan Kuhn, présentant les conséquences environnementales de l'élevage.
} 
communication, il maîtrise les concepts marketing et se trouve ainsi sur la même longueur d'onde que les entreprises qu'il cherche à convaincre (Ollitrault, 2015) :

« Les entreprises ont comme interlocuteurs des gens qui ont le même cursus qu'eux, qui leur ressemblent, parlent de la même manière, les comprennent, et ils sont pas face à un interlocuteur qui serait très associatif, qui n'aurait pas forcément le même discours, qui aurait du mal à comprendre, qui, sous prétexte de militantisme, n'arriverait pas à intégrer les exigences et les besoins de l'entreprise. Or, là, (...) on est à l'origine dans un parcours qui est classique. Donc ça les rassure. » (Pierre-Emmanuel, 40 ans, FV)

Si certains acteurs ont connu une désillusion et un désalignement de leurs valeurs dans leur vie professionnelle et estiment retrouver une cohérence entre leurs valeurs et leur environnement de travail grâce à l'engagement militant, d'autres voient dans leur expérience au sein d'une association militante une étape d'un parcours professionnel qui n'est pas envisagé exclusivement dans le champ militant :

« Je pense pas que je vais rester super longtemps [à FV]. J'avais envie de rester déjà, parce que les missions qui me sont proposées elles étaient un peu exceptionnelles, par rapport à mon âge, par rapport au fait que j'avais pas encore eu d'autre expérience. (...) Mais moi, mon idéal, c'est d'aller à Berlin et de travailler là-bas (...) sur les politiques alimentaires et l'axe durable, soutenable. » (Agathe, 24 ans, FV)

Cet extrait d'entretien souligne que ces carrières qui peuvent alterner séquences en entreprises, dans le monde associatif — voire dans le secteur public, comme le suggère ici la mention des politiques alimentaires — n'ont rien d'exceptionnelles, et constituent aujourd'hui des trajectoires professionnelles classiques. Le passage par l'action militante semble même être valorisée au sein de ces différents secteurs. Cette valorisation fait ainsi écho à l'intérêt que les associations trouvent à recruter des professionnels passés par le monde de l'entreprise. De toute évidence, ces circulations, non seulement, existent, mais semblent fortement mises en avant et appréciées tant par les individus que par les organisations, ce qui tend à les favoriser. Si ces « militants » en entreprise ont pu parfois avoir du mal à trouver leur place (Olsson, Hysing, 2019), et parfois eu besoin de mettre en retrait leurs appartenances militantes (Chiapello et Gitiaux, 2009 ; Bereni et Prud'homme, 2019), la plupart des acteurs que nous avons rencontrés 
témoignent a minima d'un intérêt ou d'une bienveillance pour les formes les plus consensuelles du militantisme végétarien, et certains affirment qu'en vendant des produits végétaux, ils accomplissent une action militante. Dans la tension entre valeurs morales et objectifs économiques de l'entreprise, certains choisissent de mettre en avant une identité orientée vers les valeurs morales (Carollo et Guerci, 2018). C'est notamment le cas d'Aurore (42 ans), responsable marketing d'une entreprise de simili-carnés et simili-poissons, qui n'hésite pas à se qualifier de « militante », tout en articulant sa démarche avec une éthique professionnelle, à la manière d'une militante professionnelle (Bereni et Prud'Homme, 2019), témoignant ainsi de la diversité des formes subjectivités militantes :

« On nous dit toujours [dans le milieu de l'agroalimentaire] : (...) 'On ne veut pas faire du militantisme'... Mais on milite, en vendant des yaourts ! (...) Moi, je suis très contente de faire faire de l'argent à ma société en vendant ce en quoi je crois. (...) J'ai des valeurs importantes, (...) je ne vends pas de produits laitiers. » (Aurore, 42 ans, responsable dans une entreprise de simili-carnés et simili-poissons)

Dans une trajectoire symétrique, partant de la démarche militante pour construire son projet professionnel, Julie (30 ans), qui gère un restaurant de burgers véganes, est fière de faire progresser la cause, par son activité économique :

« Moi je les soutiens totalement, [DA] ; c'est grâce à eux que je suis devenue végétarienne à la base, puis végane. (...) Pour moi, mon acte militant, il est déjà d'avoir ouvert ce restaurant.» (Julie, 30 ans, cofondatrice d'un fast-food végane)

Ces trajectoires similaires facilitent ainsi l'interconnaissance ou le sentiment de commune appartenance à un espace social. Autrement dit, en plus de circuler entre les mondes militants et marchands, les acteurs de cet espace ont des parcours personnels parfois mêlés. Par exemple, Pierre-Emmanuel, de FV, rencontre Alexandre - avec qui il fonde un festival végane réunissant des acteurs militants et marchands participant aux stratégies de collaboration — via la conjointe de ce dernier. Or, cette dernière est elle-même responsable dans une grande entreprise de l'agroalimentaire, dont une branche végane est active dans ces actions collaboratives. De la même façon, Pierre-Emmanuel et Agathe (FV) ont développé des liens 
cordiaux avec divers acteurs du marché de l'alimentation au cours de leurs actions. Par exemple, Agathe (FV) et Chloé (startup Veggie'z) se connaissent personnellement depuis l'introduction des produits Veggie'z dans les trains de la $\mathrm{SNCF}^{21}$ grâce au soutien de FV ; et Baptiste (de [Entreprise $\mathrm{X}]$ ) est depuis longtemps en contact avec FV puisque son entreprise est le premier groupe français de la grande distribution qui a demandé la labellisation VG-Cert à FV. Agathe nous confie d'ailleurs, hors entretien, que Baptiste est une personne " très ouverte ", avec laquelle il est agréable de travailler, suggérant aussi que les appartenances de classe soutiennent largement ce sentiment de proximité.

On comprend ainsi que la mise en œuvre des stratégies de collaboration que nous avons présentées dans la première partie sont largement facilitées par ces origines, trajectoires et circulations. Les acteurs partagent des cadres communs, et notamment l'idée selon laquelle il serait tout à fait possible d'articuler cause végétarienne et activité économique. Ils vont alors aisément trouver le moyen de faire circuler entre eux des ressources qui faciliteront la mise en œuvre des collaborations.

\section{Une circulation des ressources entre acteurs associatifs et marchands}

Le programme de labellisation et les prestations de services aux entreprises ont largement accru les ressources de l'association FV. Mais ces activités témoignent surtout de la volonté de FV de ne pas se priver des opportunités et moyens offerts par le monde économique pour développer le végétarisme. FV est associée à 13 partenaires commerciaux (entreprises et enseignes alimentaires véganes) qui contribuent directement au financement de l'association. Elle offre également des espaces de promotion à des acteurs de l'agroalimentaire dans son magazine trimestriel Solutions Veggie. Les entreprises se réjouissent de cette publicité qui leur

\footnotetext{
${ }^{21} \mathrm{FV}$ a servi d'intermédiaire entre un fournisseur de la SNCF et la marque Veggie'z. Nous revenons plus loin sur cette séquence.
} 
donne à la fois accès à une audience de consommateurs et aux ressources symboliques conférées par une association très légitime au sein de la cause :

« [FV] nous avait sollicités pour faire un petit article dans la revue (...). Et donc moi, je fais passer ça à ma collègue. Bon quand même c'est un petit tirage, c'est vraiment confidentiel... Mais, peut-être, pour la notoriété de la marque (...) c'est peut-être pas mal et (...) c'est rigolo parce qu'on (...) avait mis une pub 'galettes', et on était sur le numéro où il y avait Matthieu Ricard ${ }^{22}$ sur la première page de couv', et quand on retournait le magazine : la gamme veggie de $[\text { Entreprise } \mathrm{X}]^{23}$ ! (rire) Oh la vache ! Matthieu Ricard et la gamme veggie de [Entreprise X], pouh ! Ça, c'est du lourd. » (Baptiste, 50 ans, responsable agriculture et qualité, [Entreprise X])

Ces échanges de ressources symboliques et économiques entre FV et les entreprises constituent bien un enjeu majeur de leur collaboration, mais ils sont rendus possibles par l'existence d'un espace où les intérêts de la cause et ceux des entreprises peuvent s'articuler. Les entreprises sont ainsi prêtes à agir pour la cause végétarienne, fût-ce pour un segment seulement de leur offre, tandis que l'association ne voit pas d'inconvénient à endosser le rôle d'intermédiaire du marché et à mobiliser des catégories marchandes. Pierre-Emmanuel (FV) fait d'ailleurs un usage important des catégories des acteurs économiques quand il rappelle qu'il existe un « marché de labels concurrentiel », sur lequel il s'agit de convaincre les «clients ». FV peut même accepter un alignement de ses objectifs militants et économiques : l'association a un intérêt économique direct au développement de ce marché, puisque la redevance lui apporte des revenus. Mais elle y voit aussi un intérêt militant, puisqu'elle fait l'hypothèse que l'accroissement de l'offre tend à promouvoir l'alimentation végétarienne.

Les ressources échangées entre salariés d'associations et acteurs marchands sont aussi relationnelles. Celles-ci peuvent consister dans la mise en relation des marques avec des

\footnotetext{
${ }^{22}$ Matthieu Ricard (non anonymisé) est un moine bouddhiste tibétain, docteur en génétique cellulaire, et militant pour diverses causes humanitaires.

${ }^{23}$ La gamme 'veggie' de [Entreprise $\left.\mathrm{X}\right]$ est une marque de distributeur associée à une gamme végétarienne et végane.
} 
fournisseurs. Dans ce type d'échanges, les associations se positionnent comme marginauxsécants (Crozier et Friedberg, 1977) entre des marques qu'elles ont labellisées (ou des restaurants qu'elles ont référencés) et des fournisseurs qu'elles connaissent. FV aide ainsi les marques labellisées VG-Cert à trouver des fournisseurs dont les produits sont compatibles avec les critères de composition exigés par le label. De même, DA, en proposant aux restaurants référencés par l'initiative RestoVégé des fournisseurs, occupe cette situation d'intermédiaire. Être un intermédiaire entre des acteurs hétérogènes (fournisseurs, et marques) permet aux associations d'occuper une place nodale dans le réseau et de contrôler ce qui s'y échange, en favorisant l'expansion de l'offre végétale des marques.

Un exemple permet de saisir l'importance du rôle intermédiaire des associations véganes dans ce type d'actions. En 2018, FV propose à Colrest, un fournisseur de la SNCF, d'introduire des repas végétariens et véganes dans les TGV. Pour ce faire, l'association met en relation Colrest avec des marques véganes qu'elle connaît par ses activités de labellisation et de partenariat. Selon une ex-stagiaire à FV, ce statut de «pont » entre différents acteurs du monde marchand permet de tisser des liens durables et de collaborer avec eux sur d'autres projets, et ainsi d'atteindre de nouvelles opportunités d'influence du marché :

«Plus nous entrons en relation avec des influenceurs, plus notre action est efficace - et les entreprises sont de précieux influenceurs. Cela fait de la collaboration entre FV et la SNCF une action très efficace. (...) Cette confiance réciproque nous permet aujourd'hui de promouvoir des produits véganes, en recommandant à la SNCF de les vendre, lors de nos rencontres avec Colrest. [Par exemple], une marque française de cookies et de yaourts à boire — avec laquelle nous sommes en contact pour le développement d'une gamme végane proposée à la labellisation VG-Cert — a découvert que nous entretenions cette relation avec Colrest, et nous a demandé si nous pouvions présenter sa gamme végane à ce traiteur, dans le but de le convaincre de vendre ses produits dans les trains de la SNCF. » (Rapport de stage d'une exstagiaire à $\mathrm{FV}, 24$ ans) $)^{24}$

\footnotetext{
${ }^{24}$ Extrait initialement en anglais - notre traduction.
} 
Cet exemple suggère le rôle que l'association prend aussi au sein de l'espace économique. Cette situation est rendue possible par les trajectoires professionnelles des salariés de l'association, qui croisent celles des acteurs des entreprises, ou qui a minima s'en rapprochent. Cette proximité sociale facilite les relations de confiance qui leur permettent d'échanger des ressources économiques et symboliques. Au fond, les compétences économiques des salariés de l'association sont largement reconnues tant par cette dernière que par les entreprises elles-mêmes.

Il n'est alors pas surprenant que les acteurs partagent aussi des espaces, comme les salons ou les foires, lors desquels ils vont aussi échanger des informations, et développer les moyens de leurs collaborations. Parmi ces événements, un festival, que nous appelons ici le LOVV' Pop \& Vegan Festival constitue une véritable vitrine de l'interpénétration des espaces militant et marchand. Ce festival a lieu chaque année à Paris en septembre, depuis 2016 ; l'entrée y est gratuite et l'événement se déroule sur deux jours, durant lesquels plusieurs types de manifestations se succèdent : conférences, concerts, ateliers culinaires. Lors de l'édition 2018 du LOVV', une conférence y est organisée sur le thème de la "végéconomie » ${ }^{25}$, avec un débat réunissant quatre représentants d'entreprises partenaires de $\mathrm{FV}^{26}$, ainsi qu'Agathe, chargée de VG-Cert à FV. Tous les acteurs trouvent un intérêt à ce type de rencontres : elles constituent pour FV une occasion de faire la promotion de VG-Cert, et pour les entreprises le moyen de faire leur propre publicité à peu de frais, en s'affichant aux côtés d'une organisation militante ayant une forte influence auprès du public du festival. De plus, le LOVV' Festival se prolonge mensuellement dans des « offs », beaucoup plus confidentiels, dont l'objectif principal est de permettre à des entrepreneurs — le plus souvent, partenaires de FV — travaillant sur des

\footnotetext{
${ }^{25}$ Terme indigène renvoyant aux enjeux économiques du développement des comportements de consommation végétariens, végétaliens et véganes.

${ }^{26}$ Des entreprises avec lesquelles FV a travaillé ou auxquelles FV a attribué le label VG-Cert.
} 
projets en lien avec le végétarisme et le véganisme ${ }^{27}$ de trouver des soutiens et des financements pour de tels projets, car cet événement mensuel est fréquenté par de possibles financeurs — eux aussi, majoritairement partenaires de FV.

Cette grande porosité entre mondes marchands et mondes militants est à la fois la cause et la conséquence de ces stratégies de collaboration. Elle les rend possibles et les légitimes tant aux yeux des entreprises que de l'association, mais, dans le même temps, elle s'en trouve ellemême renforcée et légitimée. Cette collaboration pourrait probablement déboucher sur un espace de la cause au sens de Laure Bereni (2019) qui, sans les inclure dans leur intégralité, traverserait ainsi des mondes sociaux militants et économiques, en étant particulièrement façonné par les catégories économiques.

\section{Conclusion}

Nous avons voulu montrer dans cet article comment des organisations du mouvement végétarien s'engageaient dans des stratégies de collaboration avec le monde économique dans le but de faire progresser leur cause. Ces actions consistent à développer des instruments marchands, comme des référencements, des labels et des classements, mais aussi à proposer des services payants ou gratuits, pour aider des restaurants et des industriels de l'agroalimentaires à développer une offre de produits végétariens. Nous défendons que ces actions font bien partie du répertoire militant, au sens où les associations ne les déploient pas en visant des objectifs purement économiques - même si elles contribuent à accroître leurs ressources - , mais en font un usage très stratégique, qui se combine à d'autres actions plus contestataires du répertoire militant, et aux actions d'autres organisations du champ militant.

\footnotetext{
${ }^{27}$ Cela peut être le développement d'un aliment végane ou végétarien, d'un produit cosmétique végane, d'un nouvel outil de référencement de l'offre végétarienne.
} 
Le but des associations est ainsi d'orienter les pratiques des entreprises vers le développement d'un marché de l'offre végétarienne qu'elles jugent être un bon moyen de défendre leur cause.

Nous avons souligné que ces stratégies de collaboration étaient largement facilitées par l'existence d'une forte porosité entre mondes militants et mondes économiques, liée au fait que les acteurs des organisations militantes et ceux des entreprises engagées dans cette collaboration partagent des origines sociales et des trajectoires scolaires et professionnelles similaires qui permettent à la fois les circulations entre ces espaces et l'interconnaissance entre les individus. De plus, ces proximités dans les profils facilitent le partage de ressources économiques, sociales et symboliques entre des acteurs qui n'ont pas de difficulté à articuler, et parfois faire converger leurs intérêts.

Cette forme de militantisme par le marché vient compléter ce que la littérature a déjà observé sur la manière dont les acteurs militants s'appuient sur le marché pour rendre leur cause visible et effective. La spécificité du militantisme étudié ici est cependant d'engager des actions collaboratives de moyen terme avec les entreprises qui reposent sur des interactions régulières et la construction d'outils communs. Cela nous conduit à formuler deux remarques pour interroger ce militantisme par le marché. D'une part, ces stratégies de collaborations ne sont pas sans générer des tensions au sein du monde militant, dont une partie des acteurs soulignent les limites de ces actions qui ne contribuent pas toujours à faire avancer la cause, mais plutôt à la diluer dans les objectifs de la rentabilité économique. Il parait donc intéressant de considérer aussi les frictions et dissensions qui les accompagnent. D'autre part, ces actions ne sont envisageables que lorsqu'elles concernent des causes qui sont en mesure d'articuler leurs moyens d'action aux catégories du monde marchand. La cause végétarienne a su très tôt repérer les opportunités offertes par le déploiement d'une offre marchande de produits ; elle s'inscrit, de ce point de vue, dans la continuité de ses actions. Cela nous invite à ne pas considérer ce mode d'action comme un fait inédit, mais plutôt à l'explorer dans une trajectoire longue. 


\section{Bibliographie}

AgrikOLIANSKY É., 2017, «Chapitre 6 - Les “carrières militantes”. Portée et limites d'un concept narratif », dans Sociologie plurielle des comportements politiques, Paris, Presses de Sciences Po, p. 167$192,396 \mathrm{p}$.

BALSIGER P., 2014, «Between shaming corporations and promoting alternatives: The politics of an "ethical shopping map" », Journal of Consumer Culture, 14, 2, p. 218-235.

BALSIGER P., 2016, «Moral Struggles in Markets: The Fight against Battery Cages and the Rise of Cage-Free Eggs in Switzerland », European Journal of Sociology, 57, 3, p. 419-450.

BARRAL S., 2015, Capitalismes agraires: Économie politique de la grande plantation en Indonésie et en Malaisie, Paris, Presses de Sciences Po, 240 p.

BARRAUD DE LAGERIE P., 2006, «Quel consumérisme politique pour promouvoir la responsabilité sociale des entreprises? », Gestion, 31, 2, p. 119-124.

BARRAUD DE LAGERIE P., 2009, « Construire la qualité sociale : heurs et malheurs d'un projet militant », Sociologies pratiques, 18, 1, p. 121-132.

BARTLEY T., 2003, « Certifying Forests and Factories: States, Social Movements, and the Rise of Private Regulation in the Apparel and Forest Products Fields », Politics \& Society, 31, 3, p. 433-464.

BARTLEY T., 2007a, "How Foundations Shape Social Movements: The Construction of an Organizational Field and the Rise of Forest Certification », Social Problems, 54, 3, p. 229-255.

BARTLEY T., 2007b, « Institutional Emergence in an Era of Globalization: The Rise of Transnational Private Regulation of Labor and Environmental Conditions ", American Journal of Sociology, 113, 2, p. 297-351.

Bartley T., Child C., 2014, "Shaming the Corporation: The Social Production of Targets and the Anti-Sweatshop Movement », American Sociological Review, 79, 4, p. 653-679.

BERENI L., 2019, « The Women's Cause in a Field: Rethinking the Architecture of Collective Protest in the Era of Movement Institutionalization », Social Movement Studies, DOI: $\underline{10.1080 / 14742837.2019 .1679107 .}$.

BERENI L., PRUD'HOMME D., 2019, « Servir l'entreprise ou la changer ?: Les responsables diversité entre gestion, critique et performance de la vertu », Revue française de sociologie, 60, 2, p. 175-200.

BUCHTER L., 2020, « Escaping the Ellipsis of Diversity: Insider Activists' Use of Implementation Resources to Influence Organization Policy », Administrative Science Quarterly.

CALLON, M., 2017, L'emprise des marchés: Comprendre leur fonctionnement pour pouvoir les changer, Paris, La Découverte, 502 p.

CAROllo L., Guerci M., 2018, "'Activists in a Suit': Paradoxes and Metaphors in Sustainability Managers' Identity Work », Journal of Business Ethics, 148, 2, p. 249-268.

ChiAPELlO È., GitiauX F., 2009, « Les responsables développement durable des grandes entreprises. Parcours, engagement et représentations ", Revue de l'organisation responsable, 4, 1, p. 43-53.

CochOY, F., 2002, Sociologie du packaging: ou l'âne de Buridan face au marché, Paris, Presses 
Universitaires de France, 232 p.

CROZIER M., FRIEDBERG E., 2014 [1977], L'acteur et le système: les contraintes de l'action collective, Paris, Seuil, 512 p.

DubUiSSON-Quellier S., 2013, «A Market Mediation Strategy: How Social Movements Seek to Change Firms' Practices by Promoting New Principles of Product Valuation », Organization Studies, 34, 5-6, p. 683-703.

Dubuisson-Quellier S., 2016, Gouverner les conduites, Paris, Presses de Sciences Po, 480 p.

DUBUISSON-QUELLIER S., 2017, « La capture comme levier de l'intervention publique sur l'économie: Le cas de la politique publique d'affichage environnemental en France. », Revue française de sociologie, 3,3, p. 475-499.

DUBUISSON-QUELLIER S., 2018, La consommation engagée, 2e édition entièrement actualisée, Paris, Presses de Sciences Po, $154 \mathrm{p}$.

DUBUISSON-QUELLIER S., 2020, « Anti-corporate activism and market change: the role of contentious valuations », Social Movement Studies.

ESPELAND W.N., STEVens M.L., 1998, «Commensuration as a Social Process », Annual Review of Sociology, 24, 1, p. 313-343.

FILlieUle O., 2001, «Propositions pour une analyse processuelle de l'engagement individuel. Post scriptum », Revue française de science politique, 51, 1-2, p. 199-215.

FLIGSTEIN N., MCADAM D., 2011, « Toward a General Theory of Strategic Action Fields », Sociological Theory, 29, 1, p. 1-26.

GARCIA-PARPET M.-F., 2008, « La construction sociale d'un marché parfait. Le marché au cadran de Fontaines-en-Sologne », Idées économiques et sociales, 151, 1, p. 59-72.

GIROUX V., LARUE R., 2017, « Chapitre III. Les véganes », dans Le véganisme, Presses Universitaires de France, p. 66-93.

HELY M., 2004, « Les différentes formes d'entreprises associatives », Sociologies pratiques, 9, p. 27-51.

HiATT S.R., SinE W.D., TOLBERT P.S., 2009, « From Pabst to Pepsi: The Deinstitutionalization of Social Practices and the Creation of Entrepreneurial Opportunities », Administrative Science Quarterly, 54, 4, p. 635-667.

HoffmanN S., HutTer K., 2012, «Carrotmob as a New Form of Ethical Consumption. The Nature of the Concept and Avenues for Future Research », Journal of Consumer Policy, 35, 2, p. 215-236.

HONDERMARCK A., 2018, « 'La bonne digestion et l'hygiène alimentaire'. Sociologie historique d'un mouvement réformateur en faveur du végétarisme en France (1880 - 1914) », Mémoire de master de sociologie, École normale supérieure de Paris.

KARPIK L., 2000, « Le Guide rouge Michelin », Sociologie du Travail, 42, 3, p. 369-389.

KARPIK L., 2007, L'économie des singularités, Paris, Gallimard, 384 p.

King B.G., PeArCe N.A., 2010, « The Contentiousness of Markets: Politics, Social Movements, and Institutional Change in Markets », Annual Review of Sociology, 36, 1, p. 249-267. 
LerouX, B., 2015, « L'émergence de l'agriculture biologique en France : 1950-1990 ». Pour, 3, 3, p. 59-66.

LE VELLY, R., 2006, « Le commerce équitable : des échanges marchands contre et dans le marché ». Revue française de sociologie, 2, 2, p. 319-340.

LOUNSBURY M., VENTRESCA M., HIRSCH P.M., 2003, « Social movements, field frames and industry emergence: a cultural-political perspective on US recycling », Socio-Economic Review, 1, 1, p. 71-104.

MCDonnell M.-H., OdZiemKowsKa K., Pontikes E., 2021, «Bad Company: Shifts in Social Activists' Tactics and Resources After Industry Crises », Organization Science.

NEveu É., 2011, Sociologie des mouvements sociaux, Paris, La Découverte, 128 p.

Ollitrault S., 2015, Militer pour la planète: sociologie des écologistes, Rennes, Presses Universitaires de Rennes, $226 \mathrm{p}$.

OlsSON J., HYSING, E., 2019, « Inside Activism: Political Agency and Institutional Change », Oxford Research Encyclopedia of Politics.

OUEDRAOGO A., 2000, "De la secte religieuse à l'utopie philanthropique. Genèse sociale du végétarisme occidental", Annales, Histoire, Sciences Sociales, V. 55, N4, p. 825-843.

SIMONET M., 2012, "Chapitre 11 - Le monde associatif : entre travail et engagement », dans Sociologie du monde du travail, Paris, Presses Universitaires de France, p. 195-212.

STEINER P., 2005, «Le marché selon la sociologie économique », Revue européenne des sciences sociales, XLIII-132, p. 31-64.

TRAÏNI C., 2011, La cause animale (1820-1980): essai de sociologie historique, Paris, Presses Universitaires de France, 233 p.

Valiergue A., 2021, Compensation carbone : la fabrique d'un marché contesté, Paris, Sorbonne Université Presses, 262 p.

Weber K., HeInZE K.L., DeSoucey M., 2008, «Forage for Thought: Mobilizing Codes in the Movement for Grass-fed Meat and Dairy Products », Administrative Science Quarterly, 53, 3, p. 529567.

ZELIZER V.A., 1978, « Human Values and the Market: The Case of Life Insurance and Death in 19thCentury America », American Journal of Sociology, 84, 3, p. 591-610. 